\title{
Medical therapies for motor symptoms in Parkinson's Disease
}

Conrad Musey BS ${ }^{1}$, Vikram Shivkumar MD

\section{Author Affiliations:}

1. Marshall University Joan C. Edwards School of Medicine, Huntington, West Virginia

The authors have no financial disclosures to declare and no conflicts of interest to report.

\section{Corresponding Author:}

Conrad Musey BS

Marshall University Joan C. Edwards School of Medicine

Huntington, West Virginia

Email: musey@marshall.edu 


\section{Abstract}

Parkinson's disease is the second most common neurodegenerative disorder in the aging population. It is associated with progressive loss of nigrostriatal dopaminergic neurons.

Parkinsonism is a clinical syndrome that consists of motor and non-motor symptoms. It has been well described that many if not all of these deficits are associated with abnormalities related to the neurotransmitter dopamine. This review is intended for primary care physicians who often establish the diagnosis and initiate Parkinson's treatments. One challenge faced by the primary care physician is to decide the appropriate medication and starting dose. Often medications are used at very low doses or incorrect dosing schedules. This review aims to summarize these medications, their potential side effects and the rationale for medication choices.

\section{Keywords}

Parkinson's medical

\section{Introduction}

Parkinson's disease is neurodegenerative disorder. It is the second most common neurodegenerative disorder in the aging population. ${ }^{1}$ Parkinson's disease is associated with progressive loss of nigrostriatal dopaminergic neurons. ${ }^{2}$ Parkinsonism is a clinical syndrome that consists of motor symptoms such as bradykinesia (slowness of movement), akinesia (lack of movement), hypokinesia (smallness of movement), tremor, rigidity, and postural disturbance, and non-motor symptoms such as pain, sleep disturbance, apathy, depression, dementia, orthostatic hypotension and constipation. ${ }^{2}$ The symptoms of Parkinson's start on one side of the body and then spread to the other side. Cognitive impairment in Parkinson's disease is common and disabling. The prevalence of dementia is $25 \%$; in patients who live more than twenty years with motor symptoms, the prevalence rises to $83 \% .{ }^{3}$ It has been well described that many if not all of these deficits are associated with abnormalities related to the neurotransmitter dopamine. The diagnosis of Parkinson's disease is based mainly on clinical assessments. ${ }^{1}$ Parkinson's disease is diagnosed by the presence of bradykinesia and presence of rigidity, resting tremor or postural instability.

\begin{tabular}{|ll|}
\hline \multicolumn{1}{|c|}{ UK Parkinson's Disease Society Brain Bank Clinical Diagnostic Criteria } \\
\hline$\bullet$ & Bradykinesia \\
\hline$\bullet$ & Muscular rigidity \\
\hline$\bullet$ & $4-6 \mathrm{~Hz}$ rest tremor \\
\hline - & $\begin{array}{l}\text { Postural instability not caused by primary visual, vestibular, cerebellar or } \\
\text { proprioceptive dysfunction }\end{array}$ \\
\hline
\end{tabular}


This review is intended for primary care physicians who often establish the diagnosis and initiate treatments. Over the last fifty years since the advent of Levodopa, there have been multiple medications which have become available. One of the challenges faced by the primary care physician is to decide the appropriate medication and starting dose. Often medications are used at very low doses or incorrect dosing schedules. Obtaining an appointment with a movement disorders specialist in the United States often takes two to eight months which translates into inadequate symptom control for many patients. ${ }^{4}$ This review aims to summarize these medications, their potential side effects and the rationale for medication choices.

\section{Methods}

This research content is derived from a PubMed search of key words "Parkinson's" "medical treatment" and "non-surgical treatment" including citations from 1946 to January 1, 2017. The search was limited to English language articles only. This narrow range of keywords was used in an attempt to capture only articles relevant to the medical treatment of Parkinson's disease. The PubMed search strategy was developed in conjunction with the Director of Health Science Library at the Health Science Library- Joan C. Edwards School of Medicine, a librarian knowledgeable in systematic review methodology and reviewed by the investigators. The PubMed search included a focused search for "Parkinson's non-surgical treatment" without restriction to preclinical studies or age groups, followed by an expanded search for "Parkinson's medical treatment". No language or study design limits were applied. Titles and abstracts from the preliminary search were vetted to identify potentially pertinent articles. First and second authors individually assessed the full-text of these publications for eligibility and any disagreements about inclusion were agreed through discussion and consensus.

Table 1: Classification of nonsurgical Parkinson's Disease treatments

\begin{tabular}{|l|l|}
\hline Class & Medication \\
\hline \multirow{4}{*}{ Supplemental Dopamine } & Carbidopa-Levodopa IR and CR \\
\cline { 2 - 2 } & Rytary \\
\cline { 2 - 2 } & Stalevo (CD-LD + Entacapone) \\
\hline Anticholinergics & Benztropine \\
\cline { 2 - 2 } & Trihexyphenidyl \\
\hline $\begin{array}{l}\text { glutamateric N-methyl-D-aspartate } \\
\text { (NMDA) antagonist + dopamine release } \\
\text { + decreased uptake }\end{array}$ & Amantadine \\
\hline Dopamine agonists & Ropinirole \\
\cline { 2 - 2 } & Pramipexole \\
\hline & Rotigotine \\
\cline { 2 - 2 } & Apomorphine \\
\hline \multirow{2}{*}{ Monoamine Oxidase (MAO) inhibitors } & Rasagiline \\
\cline { 2 - 2 } & Selegiline \\
\hline COMT inhibitors & Entacapone \\
\cline { 2 - 2 } & Tolcapone \\
\hline
\end{tabular}

Carbidopa-Levodopa 
Currently, levodopa (LD) is the most potent medication for treatment of motor symptoms in Parkinson's disease. In 1960 Ehringer and Hornykiewicz discovered the striatal dopamine depletion in Parkinson's disease. With the knowledge that levodopa was the natural precursor to dopamine, they suggested human trials in Parkinson's disease patients. ${ }^{5}$ It is available in immediate release, controlled release and oral disintegrating forms. ${ }^{6}$ Levodopa has a relatively short half-life ( $\sim 1.5$ hours), and is quickly and extensively metabolized in plasma. ${ }^{7}$ Levodopa is actively absorbed, mainly in the proximal small intestine and is rapidly metabolized by aromatic L-amino acid decarboxylase (AADC). Therefore, levodopa products are formulated with an AADC inhibitor, commonly carbidopa (CD), to prevent peripheral metabolism and increase the fraction of levodopa transported to the brain for conversion to dopamine. ${ }^{8}$ Combining carbidopa and levodopa improves tolerability by reducing nausea and other adverse effects from peripheral decarboxylation, making oral use of levodopa practical. The ratio of carbidopa: levodopa should be ideally 1:4 for better CNS penetration. Levodopa is best absorbed if taken separate from proteins since proteins can interfere with absorption. Ideally, a gap of forty five to sixty minutes is recommended. Clinicians should initiate levodopa treatment with low doses and proceed by small increments. ${ }^{9}$ Typically, the $25 / 100$ formulation is initiated at a dose of one tab three to four times daily. Due to its short half-life, it is ideally taken at four hour intervals. Long term use of high dose of carbidopa-levodopa can cause dyskinesia, and motor fluctuations. Patients with Parkinson's disease can also develop dopamine dysregulation syndrome after many years of use. ${ }^{9}$ Carbidopa-levodopa is the preferred drug especially in the older population since it is less likely to cause side effects compared to other drugs. Most common side effects are nausea, dizziness, drowsiness, orthostasis, hallucinations and confusion. ${ }^{10}$

Controlled release (CR) formulation, which uses a degradable polymer matrix to slow the release of LD, was developed as an alternative to the Immediate Release (IR) form. It has not shown a significant reduction in "off" time as compared to IR formulation. Absorption is delayed and its effect is not as predictable. CR is mainly used at night time to help with early morning off periods. Some physicians do use it along with IR, though that does increase the complexity of dosing for patients.

\section{Anticholinergics: Benztropine, Trihexyphenidyl}

In the early 1960s, it was theorized by André Barbeau that the dopaminergic deficit would lead to an increase in striatal cholinergic activity, contributing to tremor and other symptoms. ${ }^{6}$ Anticholinergics side effects include memory loss, confusion, hallucinations, constipation, urinary retention, dry mouth, dry eyes, and blurred vision. ${ }^{6}$ There is strong evidence that the main autonomic adverse effects of cholinergic drugs are mediated by the activation of peripheral M2 and M3 receptors, while cognitive effects may be related to M1 receptor activation. ${ }^{6}$

\section{Benztropine}

Benztropine's effect is believed to be most notable for tremor ${ }^{6}$. Benztropine is initiated at 0.5 mg twice daily and increased by 0.5 to $1.0 \mathrm{mg}$ every three to four days as necessary to maximum of 3 to 4 mg per day. ${ }^{11}$ 


\section{Trihexyphenidyl}

Like benztropine, trihexyphenidyl effect is believed to be most notable for tremor. ${ }^{6}$ Trihexyphenidyl is generally initiated at $2 \mathrm{mg}$ by mouth three times a day and increased to a maximum of $5 \mathrm{mg}$ three times a day. ${ }^{11}$

\section{Amantadine}

Amantadine was developed as an antiviral agent. It was used widely in nursing home populations, and Schwab noted its unexpected benefit on tremor, balance, and akinesia in both Parkinson's disease and postencephalitic parkinsonian patients. ${ }^{5}$ Amantadine is a glutamateric Nmethyl-D-aspartate (NMDA) antagonist and has been postulated to increase dopamine release, decrease the reuptake of dopamine and stimulate dopamine receptors. ${ }^{11}$ Amantadine is often used in early Parkinson disease for tremor control and for treatment of dyskinesias and fatigue. It is the only drug that is intrinsically antidyskinetic and improves Parkinson symptoms. Side effects of amantadine include leg swelling, livedo reticularis, and neuropsychiatric and anticholinergic effects. ${ }^{12}$ Amantadine is generally initiated at $100 \mathrm{mg} / \mathrm{day}$ and increased up to $300 \mathrm{mg} / \mathrm{day}$ as necessary. ${ }^{11}$ Patients with renal failure will need dose adjustments.

\section{Dopamine agonists}

Pramipexole, ropinirole, rotigotine and apomorphine are dopamine agonists. Pramipexole and ropinirole immediate-release (IR) are rapidly absorbed and reach a maximum concentration between one and two hours after administration. ${ }^{13}$ Pramipexole and ropinirole are available in extended release (ER) forms which might be better tolerated. Their central dopaminergic adverse effects include hallucinations, psychosis, dyskinesias, dizziness, drowsiness and peripheral effects (nausea, orthostatic hypotension, edema). ${ }^{13}$ In early Parkinson's disease pramipexole and ropinirole significantly improve disability scales, delay time to dyskinesia and allow a later introduction of levodopa. Dopamine agonists are preferred as initial therapy in younger patients but might be more likely to cause side effects in the elderly. They are more likely to cause impulsive-compulsive behaviors such as gambling, shopping, hypersexuality, hoarding etc. as compared to CD-LD.

\section{Pramipexole}

Pramipexole is a full intrinsic dopamine agonist with a nonergot structure. It is a potent $\mathrm{D} 2$ agonist with maximum affinity for the D3 subtype. ${ }^{13}$ Pramipexole is available in $0.125 \mathrm{mg}, 0.25$ $\mathrm{mg}, 0.5 \mathrm{mg}, 0.75 \mathrm{mg}, 1 \mathrm{mg}, 1.5 \mathrm{mg}$ forms and is gradually titrated to target range of $1.5-4.5$ $\mathrm{mg} /$ day to be administered three times daily. ${ }^{13}$ Pramipexole tablets are taken orally, with or without food. Pramipexole has a six hour half-life ${ }^{6} .{ }^{6}$ In late Parkinson's disease, Pramipexole reduced total 'off'-time. ${ }^{13}$

\section{Ropinirole}

Ropinirole, a potent dopamine agonist, binds to both peripheral and central receptors and has maximum affinity for the D3 sub- type. ${ }^{13}$ It is dosed at $0.25,0.5,1,2$, and $5 \mathrm{mg}$. Ropinirole has 
an eight hour half-life. ${ }^{6}$ Dose up-titration is also performed on a weekly basis until reaching $8 \mathrm{mg}$ daily in the first month. ${ }^{13}$ Typical daily dose is 8 to $24 \mathrm{mg}$.

\section{Rotigotine}

Rotigotine is applied trans-dermally. Dopamine agonists are often applied as an initial treatment in Parkinson's disease due to their low potential to develop dyskinesia. ${ }^{14}$ Nowadays, mainly twenty four hour prolonged release formulations of dopamine agonists are in use and have shown significant reduction of "off" time. ${ }^{14}$ Rotigotine has a high affinity for D3 receptors as well as an agonist effect on serotonin receptors and an antagonist effect on adrenergic receptors. ${ }^{13}$ Rotigotine patches are dosed at 1, 2, 3, 4, 6, and 8 mg. ${ }^{13}$

\section{Apomorphine}

Apomorphine (APO) is a D1/D2 receptor dopamine agonist with a short half-life of nearly forty five minutes. Apomorphine is used to assess Parkinson's disease patients' responsiveness to levodopa or a dopamine agonist, as a rescue therapy for sudden off-periods. Apomorphine is also available in the UK as a continuous subcutaneous infusion for treatment of motor. ${ }^{15}$ The drug is rapidly absorbed after subcutaneous injection, and has a half-life of thirty to sixty minutes. Clinical improvements start within twenty minutes of administration. ${ }^{6}$ Apomorphine injections via pen administration are reasonable for a rapid relieve of a sudden "off" fluctuation during the daytime and nighttime and to conquer end-of-dose biphasic dyskinesia. ${ }^{14}$ Double-blind studies with injection doses of 1-5 mg have demonstrated that onset of clinical benefit typically occurs within ten minutes. ${ }^{16}$ One of the main side effects of apomorphine are skin reactions located at the injection side resulting in a formation of small nodules. Other side effects include increased daytime sleepiness, nausea, dizziness, renal impairment and orthostatic hypotension. In order to prevent nausea, the patient is pre-treated with trimethobenzamide. Neuropsychiatric changes like hallucinations and psychosis are rare but can be observed with high dosages of apomorphine. ${ }^{14}$ Apomorphine treatment is restricted only to patients with advanced Parkinson's disease due to the high cost of the drug. The long term use of APO is less likely to induce the impulsive and compulsive behaviors characterized by hypersexuality and gambling. ${ }^{15}$

\section{Selective MAO-Inhibitors}

Monoamine oxidase is a widely distributed mitochondrial enzyme. It catalyzes the oxidative deamination of a variety of monoamines, both endogenous and exogenous, and has major roles in metabolizing released neurotransmitters, and in detoxification of a large variety of endogenous and exogenous amines. ${ }^{17}$ Symptoms of Parkinson's disease are improved with dopamine antagonist (DA) replacement therapies such as DA receptor agonists and LD. Over time, however, the benefit of these drugs fluctuates and patients begin to experience loss of benefit with each dose of LD (wearing-off) and involuntary dyskinesia. In addition some parkinsonian symptoms including disturbances of gait and tremor may be resistant to dopaminergic therapy. ${ }^{17}$ Inhibition of monoamine oxidase B (MAO-B) may conserve the depleted synaptic levels of DA, and delay the need for treatment with levodopa in patients with early-stage Parkinson's disease. In patients with advanced-stage Parkinson's disease who experience fluctuations in response to 
levodopa, MAO-B inhibition potentiates and prolongs the effect of levodopa and permits use of a lower dose. ${ }^{17}$ Selegiline and rasagiline are approved as adjunct treatments to levodopa. ${ }^{11}$

\section{Selegiline}

In 1989, Tetrud and Langston published a clinical study based on the discovery that selegiline blocked the development of MPTP-induced parkinsonism in laboratory animals. ${ }^{17}$ Selegiline undergoes first-pass metabolism to $\mathrm{R}(-)$ amphetamine and $\mathrm{R}(-)$ methamphetamine, which have the potential to cause cardiovascular and CNS adverse effects. ${ }^{17}$ Selegiline is dosed at 5 to 10 $\mathrm{mg} /$ day. ${ }^{18}$ In general, selegiline is well tolerated. Side effects/adverse effects like nausea, vomiting, sleeplessness, dry mouth, orthostatic hypotension and dyskinesias have all been observed in the range of 2-5\% of Parkinson's disease patients. Other side effects like headaches, palpitations, dyspnea, confusion, edema, micturition dysfunction, loss of appetite and anxiety have an incidence below $2 \%$.

\section{Rasagiline}

Rasagiline is a potent, selective, irreversible inhibitor of MAO-B and in contrast to selegiline has no amphetamine-like metabolites. ${ }^{17}$ Rasagiline shows good antiparkinsonian and motor restoration activity as well as neuroprotection properties. ${ }^{17}$ Rasagiline is dosed at $1 \mathrm{mg} /$ day. ${ }^{18}$ Side effects of rasagiline include nausea, vomiting, orthostatic hypotension, somnolence, hallucinations and dyskinesias. ${ }^{18}$ Because rasagiline inhibits MAO-B more potently than selegiline it has the advantage of once-daily dosing. ${ }^{19}$ Rasagiline has demonstrated neuroprotective properties in a host of in vitro and in vivo studies. The ADAGIO (Attenuation of Disease Progression with Azilect Given Once-daily) trial suggested potential disease-modifying effects for rasagiline at $1 \mathrm{mg} / \mathrm{day}$, though no such benefit was observed at $2 \mathrm{mg} /$ day doses. The study also showed that it is safe to use with selective serotonin reuptake inhibitor. Patients with Parkinson's disease often have depression or anxiety, both of which can impair quality of life. ${ }^{19}$ Rasagiline at a dose of $1 \mathrm{mg}$ /day slowed the rate of Unified Parkinson's disease Rating Scale (UPDRS) progression, improved symptoms from baseline and was shown to be non-inferior. ${ }^{19}$

\section{COMT Inhibitors}

Catechol-O-methyltransferase (COMT) is an enzyme that degrades catecholamines like dopamine. Entacapone and tolcapone are COMT inhibitors. They are another adjunctive option for the treatment of advanced Parkinson's disease. The enzyme COMT breaks down levodopa; therefore, the addition of a COMT inhibitor allows more levodopa to reach the brain. ${ }^{11}$ Entacapone acts peripherally while tolcapone acts peripherally and centrally.

\section{Entacapone}

Entacapone is effective in reducing "off" time at an average of 0.7 to 1.2 hours per day and should be offered to patients with motor fluctuations. Entacapone is available in $200 \mathrm{mg}$ tablets. One tablet is given with each dose of levodopa, not to exceed a total daily dose of $1600 \mathrm{mg} .{ }^{11}$ The most common adverse effects of entacapone include dyskinesia, nausea, diarrhea, and urine 
discoloration. ${ }^{20}$ Stalevo is a formulation that contains combination of carbidopa, levodopa and entacapone.

\section{Tolcapone}

Tolcapone has been determined to be probably effective in the treatment of "off" time with an average daily reduction in "off" time of 0.9 to 1.8 hours. ${ }^{11}$ Tolcapone is initiated at $100 \mathrm{mg}$ three times daily and increased to $200 \mathrm{mg}$ three times daily if necessary. ${ }^{11}$ Tolcapone arrived on the market in 1997/1998 and its side effects include elevated liver transaminases and hepatotoxicity. $^{21}$

\section{Rytary}

Levodopa is the most effective oral pharmacotherapy developed to date and is typically administered in combination with a dopa-decarboxylase inhibitor such as carbidopa. Rytary is an extended-release (ER) formulation of carbidopa and levodopa in a 1:4 ratio. It was designed to rapidly achieve therapeutic plasma levodopa concentrations and maintain them for a prolonged duration to allow a dosing interval of approximately 6 hours in both early and advanced disease. ${ }^{22}$ The most commonly reported adverse events with Rytary included nausea, dizziness, and headache. ${ }^{23}$ In early Parkinson's disease, the most frequently reported adverse effects, nausea and insomnia, are commonly reported for dopaminergic drugs. In advanced Parkinson's disease, the most frequently reported adverse effects, dyskinesia and fall, are commonly associated with Parkinson's disease therapies and/or Parkinson's disease progression. ${ }^{22}$ The ADVANCE-PD study showed that patients can be safely switched from standard immediate release (IR) CD-LD to rytary with reduced "off", decreased fluctuations, and better quality of life. ASCEND- PD study reported that rytary significantly decreased "off" time and increased "on" time without troublesome dyskinesia, as compared to stalevo (CD-LD-entacapone). ${ }^{7}$ Immediate release carbidopa-levodopa has a short half-life. Over time, there is shortening of clinical response and higher more frequent dosing is needed. It is believed that the short interval dosing causes post synaptic changes in the striatum. These changes, as well as progression of the disease, lead to motor fluctuations and dyskinesias. Patients who are experiencing motor fluctuations are probably the best candidates for rytary. ${ }^{7}$ In order to transition a patient from regular carbidopa/levodopa to rytary, the total daily levodopa dose is roughly doubled and divided into three or four doses.

Management of motor symptoms in Parkinson's disease is very complex. Medications with different mechanisms of action are often used in conjunction. Contrary to general principles, Parkinson's disease is a condition in which we often use polypharmacy to achieve better outcomes. To summarize, younger patients ( $<65-70$ years) benefit from dopamine agonists or MAO-B inhibitors to begin with as this delays use of levodopa and the subsequent onset of motor fluctuations. However, younger patients who do not respond adequately to other agents should be started on levodopa. Older patients tend to tolerate levodopa better than other agents and it should, therefore, be the drug of choice. Amantadine and anticholinergics are particularly beneficial in treating tremor. The landscape of medical therapies for motor symptoms in Parkinson's disease continues to expand. Some of the ones in the pipeline include the accordian pill, inhaled levodopa, tozadenant, sublingual apomorphine. Appropriate choice of medications will provide greater symptom relief for patients prior to seeing a specialist. 


\section{References}

1. Gao LL, Wu T. The study of brain functional connectivity in Parkinson's disease. Transl Neurodegener. 2016;5:18.

2. Nishijima $\mathrm{H}$, Tomiyama M. What mechanisms are responsible for the reuptake of levodopa-derived dopamine in parkinsonian striatum? Front Neurosci. 2016;10:575.

3. Leaver K, Poston KL. Do CSF biomarkers predict progression to cognitive impairment in Parkinson's disease patients? A systematic review. Neuropsychol Rev. 2015;25(4):411-23.

4. Holmes K et al. How long is the wait to see a specialist in Parkinson's disease in the United States? (S19.003). Neurology. 2016;86(16 Supplement).

5. Goetz CG. The history of Parkinson's disease: early clinical descriptions and neurological therapies. Cold Spring Harb Perspect Med. 2011;1(1):a008862.

6. Smith Yet al. Parkinson's disease therapeutics: new developments and challenges since the introduction of levodopa. Neuropsychopharmacology. 2012;37(1):213-46.

7. Dhall R, Kreitzman DL. Advances in levodopa therapy for Parkinson disease: Review of RYTARY (carbidopa and levodopa) clinical efficacy and safety. Neurology. 2016;86(14 Suppl 1):S13-24.

8. Hsu A et al. Comparison of the pharmacokinetics of an oral extended-release capsule formulation of carbidopa-levodopa (IPX066) with immediate-release carbidopa-levodopa (Sinemet((R))), sustainedrelease carbidopa-levodopa (Sinemet((R)) CR), and carbidopa-levodopa-entacapone (Stalevo((R))). J Clin Pharmacol. 2015;55(9):995-1003.

9. Ferrazzoli D et al. Dopamine replacement therapy, learning and reward prediction in Parkinson's disease: implications for rehabilitation. Front Behav Neurosci. 2016;10:121.

10. Brodell DW et al. Carbidopa/levodopa dose elevation and safety concerns in Parkinson's patients: a crosssectional and cohort design. BMJ Open. 2012; 2(6).

11. Nashatizadeh MM, Lyons KE, Pahwa R. A review of ropinirole prolonged release in Parkinson's disease. Clin Interv Aging. 2009;4:179-86.

12. Fernandez HH. 2015 update on Parkinson disease. Cleve Clin J Med. 2015;82(9):563-8.

13. Alonso Canovas A et al. Dopaminergic agonists in Parkinson's disease. Neurologia. 2014;29(4):230-41.

14. Ossig C, Reichmann H. Treatment of Parkinson's disease in the advanced stage. J Neural Transm (Vienna). 2013;120(4):523-9.

15. Ribaric S.The pharmacological properties and therapeutic use of apomorphine. Molecules. 2012;17(5): 5289-309.

16. Factor SA. Literature review: intermittent subcutaneous apomorphine therapy in Parkinson's disease. Neurology. 2004;62(6 Suppl 4):S12-7.

17. Finberg JP, Rabey JM. Inhibitors of MAO-A and MAO-B in psychiatry and neurology. Front Pharmacol. 2016;7:340.

18. Riederer P, Laux G. MAO-inhibitors in Parkinson's disease. Exp Neurobiol. 2011;20(1):1-17.

19. Leegwater-Kim J, Bortan E. The role of rasagiline in the treatment of Parkinson's disease. Clin Interv Aging. 2010;5:149-56.

20. Chong BS, Mersfelder TL. Entacapone. Ann Pharmacother. 2000;34(9):1056-65.

21. Truong DD. Tolcapone: review of its pharmacology and use as adjunctive therapy in patients with Parkinson's disease. Clin Interv Aging. 2009;4:109-13.

22. Waters $\mathrm{CH}$ et al. Long-term treatment with extended-release carbidopa-levodopa (IPX066) in early and advanced Parkinson's disease: A 9-month open-label extension trial. CNS Drugs. 2015;29(4): 341-50.

23. Pahwa R et al. Randomized trial of IPX066, carbidopa/levodopa extended release, in early Parkinson's disease. Parkinsonism Relat Disord. 2014;20(2):142-8. 